\title{
Differential RNA editing of ATP complex in different tissues of Catharanthus roseus plastid
}

\author{
Wasimah B Alshammari 1, 2, Huda Alhamdan 1, Thana Khan 1, Sameh E. Hassanein 4, 5, Ahmed M \\ Ramadan 1,6 \\ ${ }^{1}$ Department of Biological Sciences, Faculty of Science, King Abdulaziz University, Jeddah, Saudi Arabia \\ ${ }^{2}$ Department of biology, faculty of science, Hail University, Hail, Saudi Arabia \\ ${ }^{4}$ Bioinformatics and Computer Networks Department, Agricultural Genetic Engineering Research Institute (AGERI), Agriculture Research Center (ARC), Giza, \\ Egypt \\ ${ }^{5}$ College of Biotechnology, Misr University for Science and Technology (MUST), 6th October city, Egypt \\ ${ }^{6}$ Agricultural Genetic Engineering Research Institute (AGERI), Agriculture Research Center (ARC), Giza, Egypt
}

Correspondence Author: Wasimah B Alshammari, Department of Biological Sciences, Faculty of Science, King Abdulaziz University, Jeddah, Saudi Arabia

2Department of biology, faculty of science, Hail University, Hail, Saudi Arabia

Email: alshamarywasima@yahoo.com

Received date: 12 December 2019, Accepted date: 28 January 2020, Online date: 31 January 2020

Copyright: (C) 2020 Wasimah B Alshammari et al., This is an open-access article distributed under the terms of the Creative Commons Attribution License, which permits unrestricted use, distribution, and reproduction in any medium, provided the original author and source are credited.

\begin{abstract}
Individual nucleotides in RNA transcripts of plant organelles undergoes a post-transcriptional alteration known as RNA editing which can lead to the divergence of amino acids encoded by edited RNA from the proposed products. In this study, RNA editing sites of protein-coding atp gene complex from the plastid genome of Catharanthus roseus was analysis and then compared across cDNA transcripts of different tissues (flower, leaf, stem, root, hairy root and seedling). C-to-U editing sites were identified via bioinformatic tool which led to 4 amino acids changes. In total 5 editing sites, 4 affected deduced amino acids and one was silent, were predicted in only two genes out of six. Three editing sites in atpA (C737, C791, C914) and two in atpI (C438, 620) The five edited sites were distributed differently among the six tissues informing that RNA editing in plastid is tissue-dependent. Interesting, editing events occurred at the same quantity in leaves, stems, roots, hairy roots and seedlings and slight fewer in flower tissues. All four affected deduced amino acids led to the increase of the hydrophobicity of $\operatorname{atpA}$ and $a t p I$ proteins indicating that RNA editing might have an influence on the function of the target genes. The current study may provide important input for detecting the strength of RNA editing in the ATP complex of different organs.
\end{abstract}

Keywords: Catharanthus roseus, RNA editing, chloroplast, ATP complex

\section{INTRODUCTION}

RNA editing is defined as the action by which RNA sequence nucleotides subject to modulations, such as conversions or additions/deletions thus it changes the influx of genetic details from the DNA to grown RNA [1]. Various types of RNA editing events and mechanisms have been documented in viruses, primitive eukaryotes, vertebrates, fungi, and plants. C-to-U and A-to-I conversions are widespread editing events in animals and plants [2]. The RNA editing apparatus in plant together referred to as the editosome which composed of a minimum of four protein groups including PPRs (pentatricopeptide repeat) protein, MORFs (multiple organellar RNA editing factors) /RIPs (RNA editing factor interacting proteins), ORRM1 (organelle RNA recognition motif) and OZ1 (organelle zinc-finger) [3]. The PPR gene group is one of the biggest gene classes in plants especially in Arabidopsis, involving more than 400 genes [4] which control the RNA editing process either immediately or indirectly [5, 6, 7]. In addition, the MORF/RIP, ARM and OZ proteins are engaged in RNA editing directly or indirectly and play a key function in the editosome complex [3]. These data point out that RNA editing status is a natural physiographic event in plant organelles.

The DYW domain in the PPR protein family, which displays C-to-U substitution reaction, has been described to have a similar action as the cytidine deaminase protein [8]. The specialized PPR proteins are a type of protein that recognizes and binds to their related RNA [11]. The efficiency of RNA editing diverges with the organ type and stage of development; for example, RNA editing is more prevalent in photosynthesis organs than in non-photosynthesis organs [13, 12]. It has been proposed that RNA 
editing is evolutionary practicability, which has not noticed in rudimentary organisms and it can adapt organisms and fix DNA damage caused by external factors $[1,14]$. Moreover, RNA editing often tends to substitute amino acids from hydrophilic to hydrophobic residues which are a substantial trait of protein-protein interfaces that control stabilization and induce the enzymatic activity of protein [15].

RNA editing documented mostly in mitochondria and plastid organelles in almost all species of land plants. The chloroplasts are the type of plastids present merely in plant and algae cells that carry out photosynthesis and biochemical path [16]. They contain their own DNA encoding over 100 proteins indispensable for cellular processes such as photosynthetic reactions and other major organelle tasks [17]. The chloroplast adenosine triphosphate (ATP) synthase genes complex is one of the important genes encoding proteins implicated in the photosynthesis process. It composes of two major sub-complexes, FO and F1 [18]. The F0 group functions in proton transport across the membrane and comprises of a membrane-intrinsic c-ring subunit (AtpH) and 3 membrane-intrinsic subunits, containing AtpF, AtpG, and AtpI. The CF1 group has 5 subunits including AtpA, AtpB, AtpC, AtpD and $A t p E$ [19]. The plastid ATPase subunits are encoded by both the nuclear and organellar genomes where the subunits atpA, $a t p B, a t p F, a t p H, a t p E$ and $a t p I$ are encoded in the plastid [20].

In the present study, we tested RNA editing in atp complex in various tissues (flower, leaf, stem, root, hairy root, and seedling) of Catharanthus roseus plastid (accession no. KC561139.1). Using bioinformatics tool editing events and deduced amino acids were further compared across the six different tissues. Our data detected tissue-dependent RNA editing sites. The details obtained in this study will enhance our conception of RNA editing in ATP complex.

\section{MATERIALS AND METHODS}

\section{1- Database for atp complex in $C$. roseus plastid and RNA -seq}

ATP genes complex in $C$. roseus plastid was downloaded from Gene Bank at the National Center for Biotechnology Information (NCBI) (https://www.ncbi.nlm.nih.gov/nuccore/KC561139.1/). Also, RNA-Seq in C. roseus plastid tissues (flowers, leaves, stems, roots, hairy roots and seedlings) was gathered from the Sequence Read Archive (SRA) database in NCBI. Accession number of $C$. roseus chloroplast atp genes complex was (KC561139.1), Accession numbers of RNA-Seq as follow: flower (SRR122239), leaf (SRR122251), stem (SRR122253), root (SRR122254), hairy root (SRR122257), seedling (SRR122243).

\section{2- Bioinformatics Analysis of RNA Editing Events}

ATP genes and RNA-Seq transcripts from the six tissues of C. roseus plastid used in the present study were analyzed for RNA editing sites. A multi-sequence alignment by CLC genomic workbench 3.6.5, software web server (http://www.clcbio.com/products/clc-genomics-workbench) was used to identify RNA editing sites in these transcripts. Also, multi -sequence alignments using the same program was applied to compare edited sites among the different tissues.

\section{3- Analysis of Amino Acids Deduced by RNA Editing}

We mapped the RNA-Seq reads to the plastid atp genes of $C$. roseus using multi-sequence alignment by CLC genomic workbench 3.6.5. We conducted a comparison of amino acid sequences among RNA transcripts of different tissues (flower, leaf, stem, root, hairy root and seedling) and the altered amino acids induced by editing were analyzed to inspect the effect of RNA editing on the final products.

\section{4- Accession Numbers of ATP transcripts in the six tissues}

RNA Sequences from this article have been submitted to GenBank data library under accession numbers: atpA in flower (MN987561), atpA in leaf (MN987562), atpA in stem (MN987563), atpA in root (MN987564), atpA in hairy root (MN987565), atpA in seedling (MN987566), atpI in flower (MN987567), atpI in leaf (MN987568), atpI in stem (MN987569), atpI in root (MN987570), atpI in hairy root (MN987571), atpI in seedling (MN987572), atpB in flower (MN987573), atpB in leaf (MN987574), atpB in stem (MN987575), atpB in root (MN987576), atpB in hairy root (MN987577), atpB in seedling (MN987578), atpE in flower (MN987579), atpB in leaf (MN987580), atpE in stem (MN987581), atpE in root (MN987582), atpE in hairy root (MN987583), atpE in seedling (MN987584), atpF in flower (MN987585), atpF in leaf (MN987586), atpF in stem (MN987587), atpF in root (MN987588), atpF in hairy root (MN987589), atpF in seedling (MN987590), atpH in flower (MN987591), atpH in leaf (MN987592), atpH in stem (MN987593), atpH in root (MN987594), atpH in hairy root (MN987595), atpH in seedling (MN987596).

\section{RESULTS}

\section{ATP complex characterization in $C$. roseus.}

ATP genes group in plastid contains six genes atpA, atpB, atpE, atpF, atpH and atpI. The size of these genes are 1518 bp, 1488 bp, 399 bp, 570, 246 and 738 bp respectively. Genes of atp complex and mRNA transcripts of flower, leaf, stem, root, hairy root and seedling tissues were collected. In total, 89,191,351 paired flower RNA reads, 122,063,131 paired leaf RNA reads, 44,283,997 paired stem RNA reads, 85,884,081 paired root RNA reads, 75,338,016 paired hairy root RNA reads, and $74,297,533$ paired seedlings were assembled to the plastid atp genes (used as references) under accession no. (KC561139.1) to recognize RNA status. Further, mRNA transcripts across the different tissues were aligned with genes references to detect editing sites and then examine the modification at amino acid level induced by editing. 


\section{Overall RNA editing identity in ATP transcripts of $C$. roseus}

In general, the alignment of mRNA transcripts among the six tissues of $C$. roseus (flower, leaf, stem, root, hairy root and seedling) with the references revealed five C-T editing sites. Edited sites were found in two of six transcripts; 3 sites in atpA (737, 791 and 914) (Fig.1) and 2 in atpI (438 and 620) (Fig.2). The remaining genes have no editing events in atpB, atpE, atpF and $\operatorname{atpH}$. The four edited sites atpA-737, atpA-791, atpA-914 and atpI- 620 altered the deduced amino acids whereas one editing site that cannot convert amino acid type was silent in atpI-438.

Bioinformatics tool showed that editing in atpA-737 and atpA-791 sites were present in all examined tissues (Table 1 and Fig. 1). Edited site atpA-914 was found in all tissues except in flower (Table 1 and Fig. 1). Also, editing sites in atpI-438 and atpI-620 were found in all six tissues (Table 1 and Fig.2). Furthermore, RNA editing primarily occurred in the second codon position where 4 out of 5 editing events were detected in the second position and one in the third location of the codon.

Table 1: A comparison of RNA-Editing Sites in plastid atpA and atpI genes among six tissues of $C$. roseus. E (edit), N (no editing)

\begin{tabular}{|c|c|c|c|c|c|c|c|c|}
\hline \multirow{2}{*}{ Gene } & \multirow{2}{*}{ Site Position } & \multirow{2}{*}{ Amino Acid Alteration } & \multicolumn{6}{|c|}{ Tissues type } \\
\cline { 4 - 9 } & & Slower & Leaf & Stem & Root & Hairy root & Seedling \\
\hline \multirow{3}{*}{ atpA } & 773 & Set-Leu & E & E & E & E & E & E \\
\cline { 2 - 9 } & 791 & Set-Leu & E & E & E & E & E & E \\
\cline { 2 - 9 } & 914 & Phe-Phe & E & E & E & E & E & E \\
\hline \multirow{2}{*}{ atpI } & 438 & Set-Leu & E & E & E & E & E & E \\
\cline { 2 - 9 } & 620 & & & & &
\end{tabular}

Table 2: A comparison of amino acids substitutions in plastid atpA and atpI among six tissues of C. roseus

\begin{tabular}{|c|c|c|c|c|c|}
\hline \multirow{2}{*}{ Tissue } & \multicolumn{4}{|c|}{ Codon Position of Amino Acid Conversions } \\
\cline { 2 - 6 } & \multicolumn{3}{|c|}{ atpA } & \multicolumn{2}{c|}{ atpI } \\
\cline { 2 - 6 } & $\mathbf{7 7 3}$ & $\mathbf{7 9 1}$ & $\mathbf{9 1 4}$ & N38 & $\mathbf{6 2 0}$ \\
\hline Flower & Ser-Leu & Pro-Leu & No & No change & Ser-Leu \\
\hline Leaf & Ser-Leu & Pro-Leu & Ser-Leu & No change & Ser-Leu \\
\hline Stem & Ser-Leu & Pro-Leu & Ser-Leu & No change & Ser-Leu \\
\hline Root & Ser-Leu & Pro-Leu & Ser-Leu & No change & Ser-Leu \\
\hline Hairy Root & Ser-Leu & Pro-Leu & Ser-Leu & No change & Ser-Leu \\
\hline Seedling & Ser-Leu & Pro-Leu & Ser-Leu & No change & Ser-Leu \\
\hline
\end{tabular}

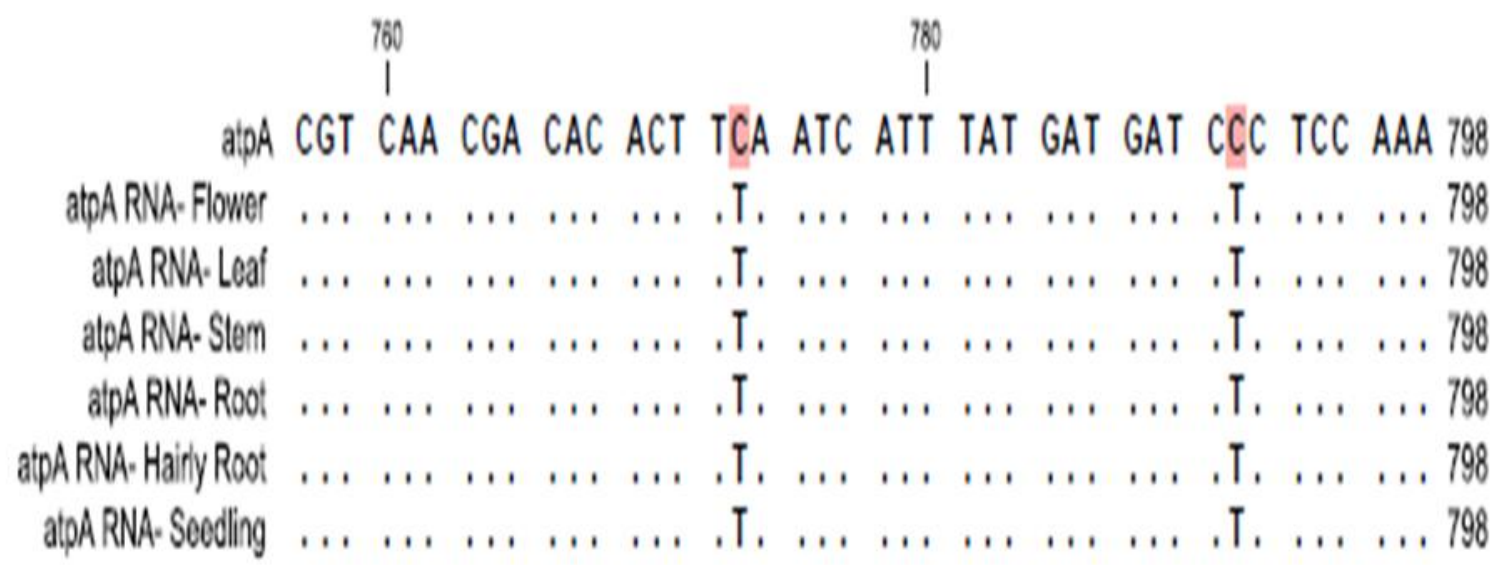




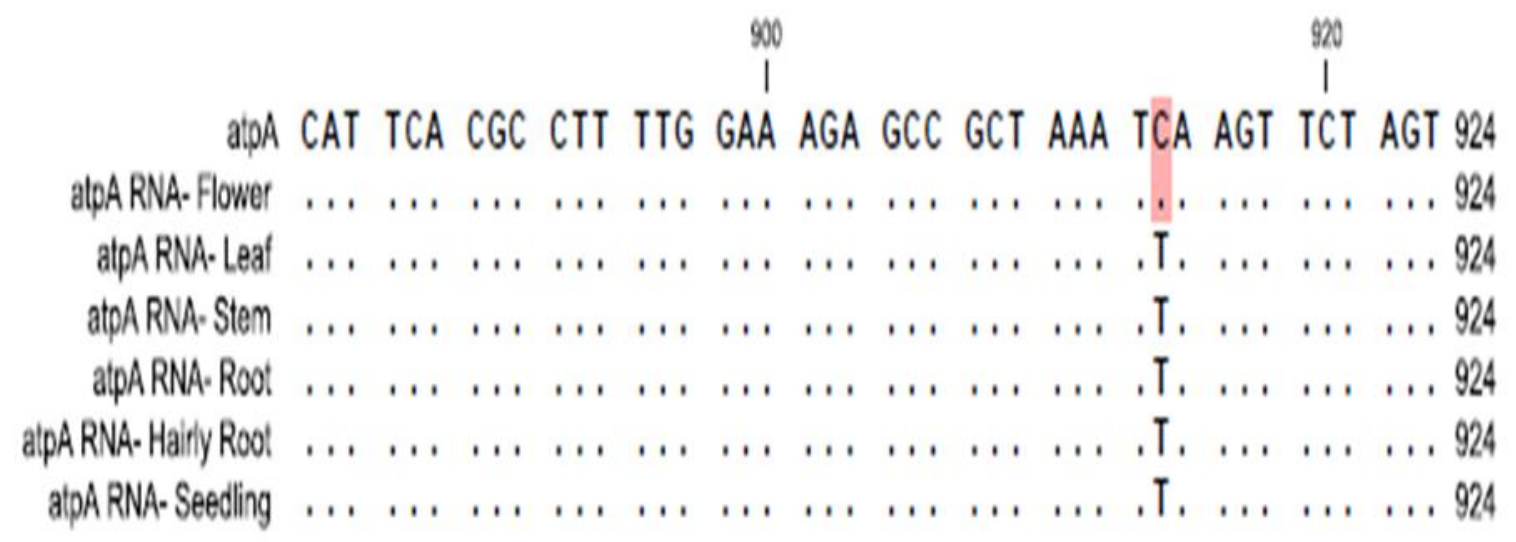

Fig. 1: A Multiple Alignment of editing sites in atpA transcripts across different tissues of Catharanthus roseus.

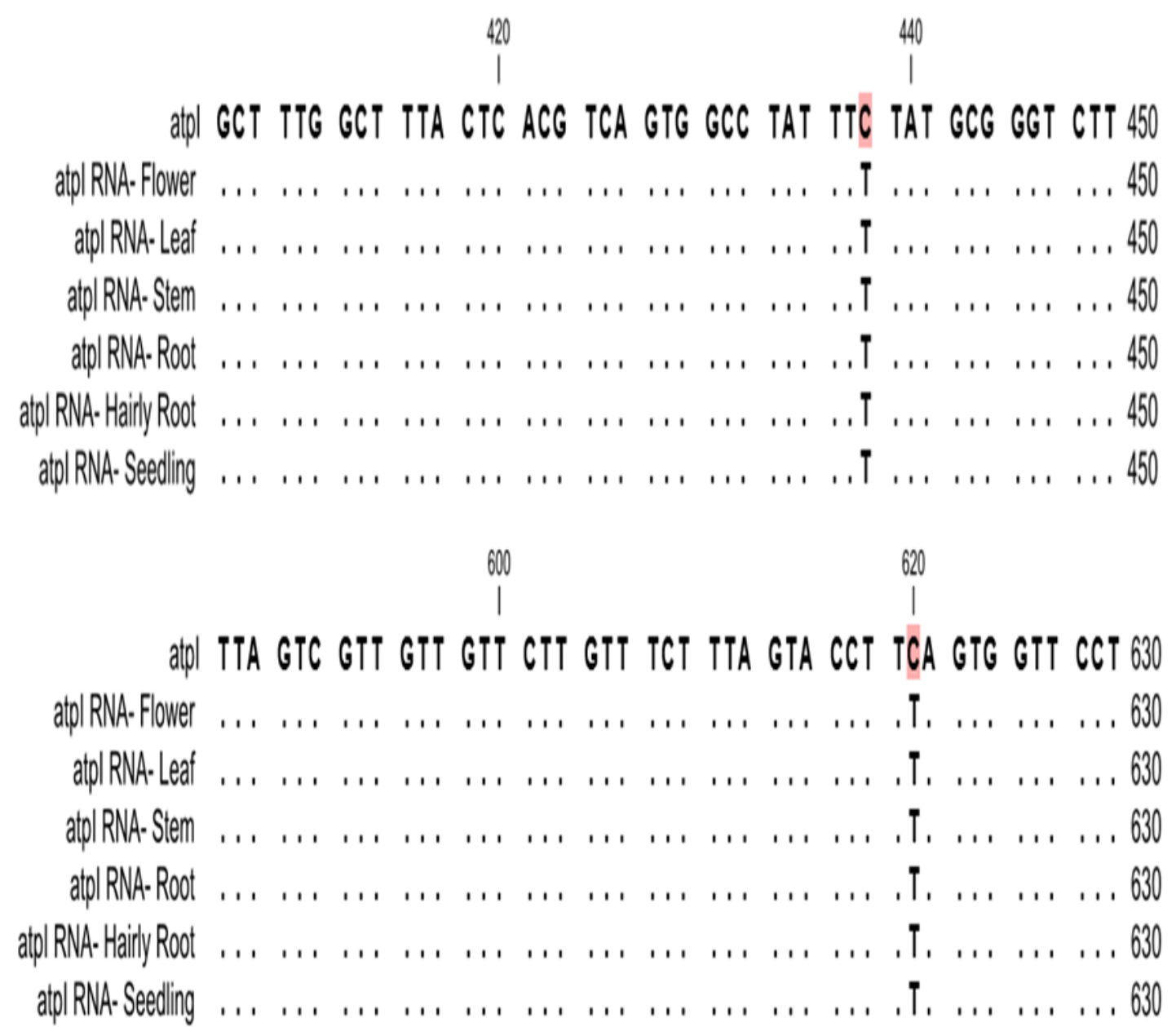

Fig. 2: A Multiple Alignment of editing sites in atpI transcripts across different tissues of Catharanthus roseus.

\section{Analyses of the eventual amino acids produced by RNA editing among the different tissues}

Further, all five converted amino acids found in atp genes of $C$. roseus were analyzed. Most of RNA editing sites occur in the protein-coding region and often leads to amino acid alterations. Of the five edited sites in ATP genes (atpA-737 atpA-791, atpA914 and atpI-620) excluding atpI-438 which is a synonym editing site with no amino acid effect, four sites caused codons to convert (fig. 3 and 4). All four sites switched amino acids from hydrophilic to hydrophobic. Interestingly, all four events emphasis the changing of amino acids to leucine (fig. 3 and 4). Besides, all five converted amino acids in atpA and atpI were observed in all tissue types except in atpI-438 was not detected in flower. 


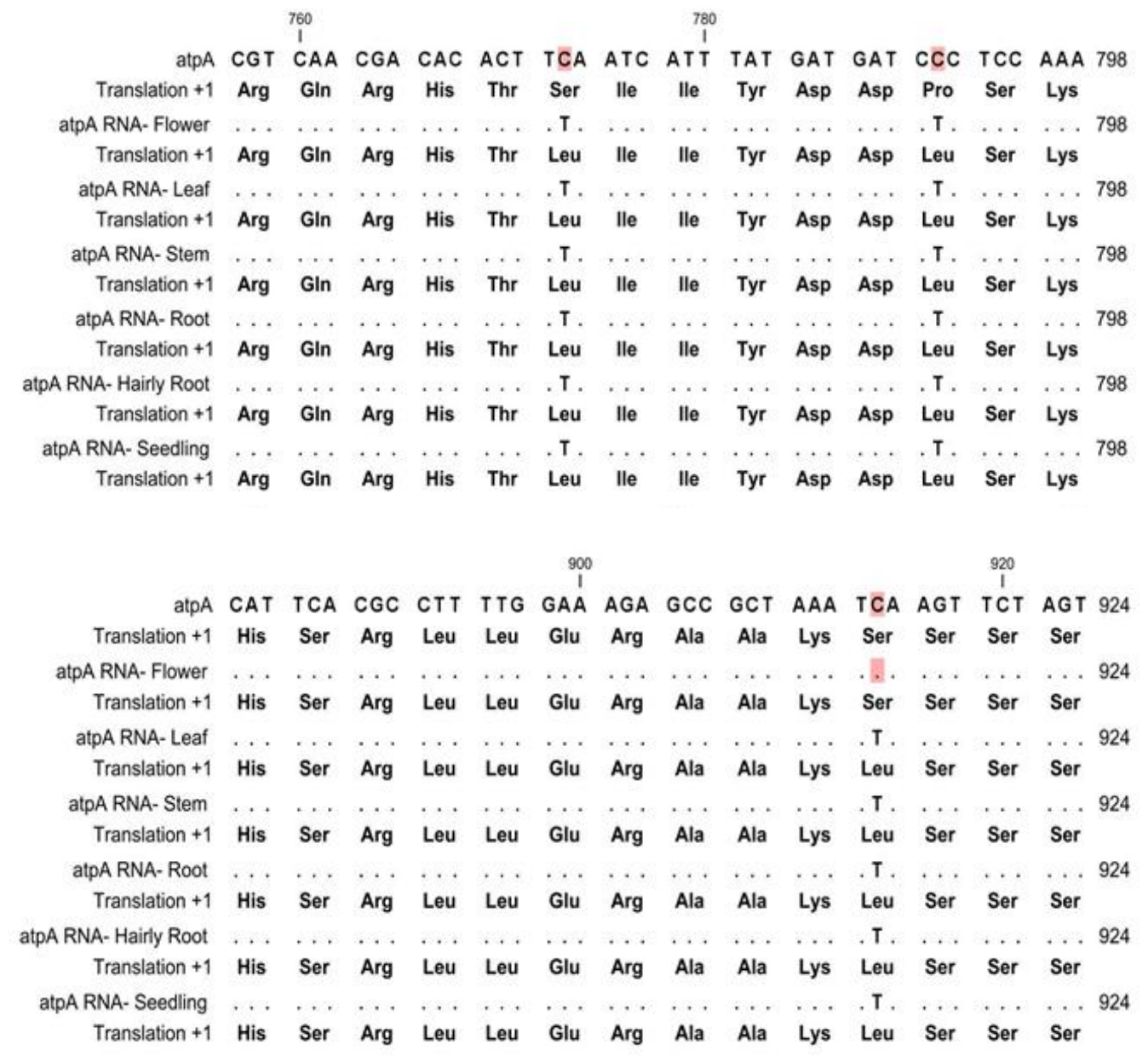

Fig. 3: A Multiple Alignment of amino acid conversions in atpA transcripts across different tissues of Catharanthus roseus.

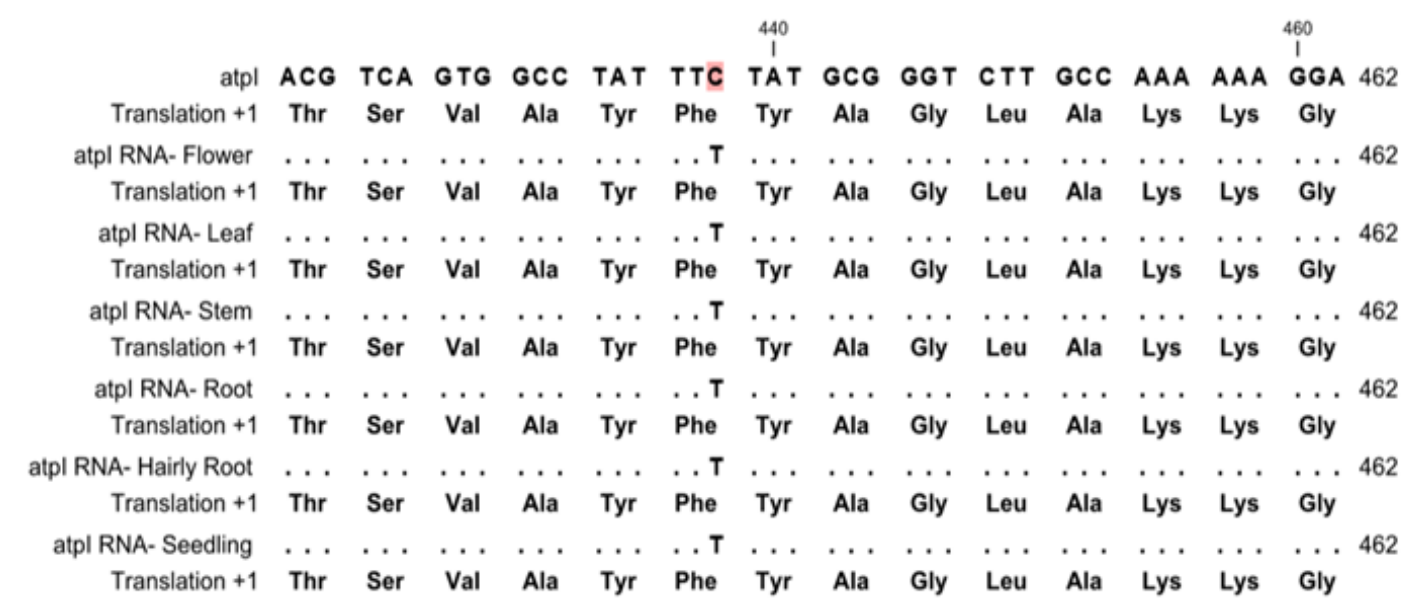




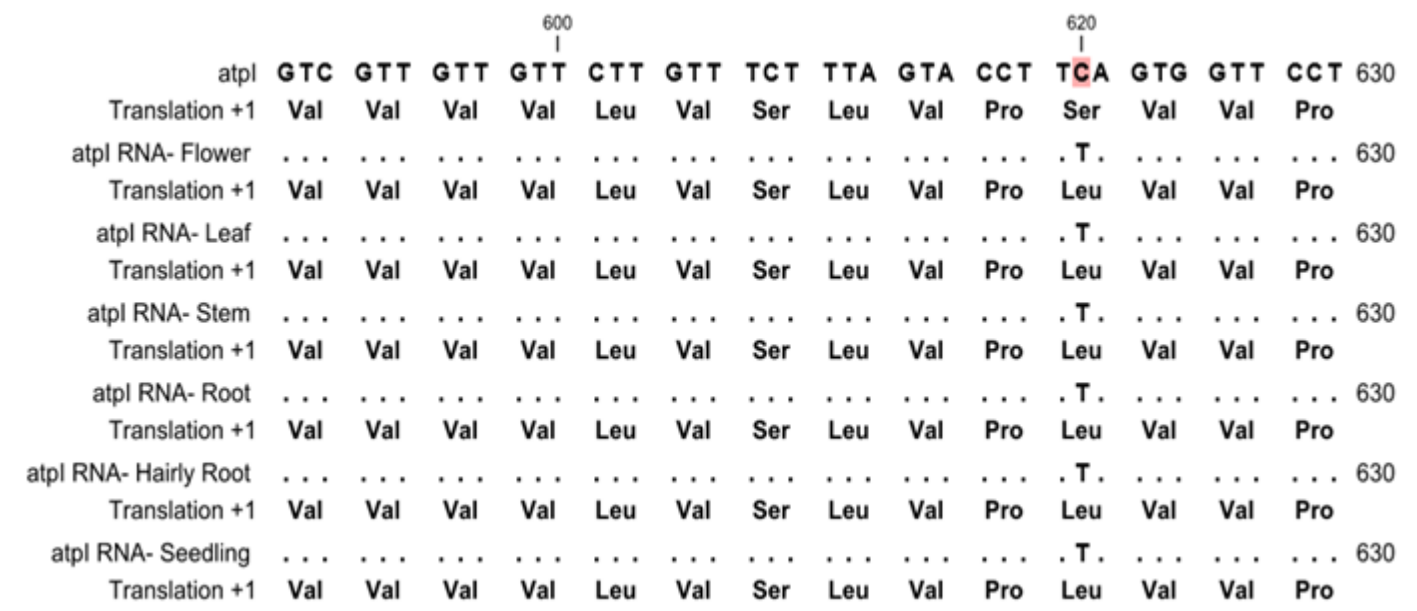

Fig. 4: A Multiple Alignment of amino acid conversions in atpI transcripts across different tissues of Catharanthus roseus.

\section{DISCUSSION}

RNA editing is a biological event occurs in plant organelles to switch cytidine to uridine resulting in the change of RNA sequences [35]. The primary function of chloroplast RNA editing is to produce codons important for protein activity [21]. Plastid ATP synthase is a thylakoid membrane-linked photosynthetic complex involved in the light-dependent reactions of the photosynthesis process [11]. The chloroplast atp genes (ATP synthase complex) encodes six subunits atpA, atpB, atpE, atpF, atpH and atpI in thylakoid membrane [20]. Here, we examined RNA editing in plastid atp complex transcripts of C. roseus in six different tissues (flower, leaf, stem, root, hairy root and seedling).

In this study, five editing sites were predicted; four caused amino acid conversions and one was silent. Findings showed that two of the atp genes (atpA and atpI) submitted to RNA editing which makes the manufactured amino acids more conserved and hydrophobic and four transcripts remained unaltered.

Analyzing of both atpA and atpI edited transcripts in the six tissues showed editing events in all tissues except atpA-914 transcript in flower has no editing (fig. 1 and 2). All tissues have the same amount of RNA editing events at atpA transcripts with three edited sites excluding in flower that have only 2 edited sites where editing at atpA-914 site was absent (fig. 1). All these editing events took place in a functional coding region, which may indicate that RNA editing could influence the structure and function of induced amino acids by editing.

Unnecessary editing sites are gone, whereas the vital sites are preserved [15]. All five detected edited sites in both atpA and atpI were conservative compared with editing sites in other plant species. C. roses atp complex shares 2 editing sites atpA-791and atpI-620 with cotton and one site atpA-914 with cotton and coconut [21]. Moreover, atpA-791 site was conserved with Arabidopsis, tobacco, pea, wheat and rice [25, 26, 27, 28, and 29], atpA-773 site with black pine and aphrodite- orchid, and atpI438 with maize [24]. These analyses suggest that Catharanthus has a close linkage with cotton regarding to atp edited sites. RNA editing events in catharanthus atpA and atpI mostly regain the conserved amino acid among higher plant species, which thus confirms that editing at RNA level is a transcription reparation mechanism [31]. A comparative analysis of editing sites across the various tissues after editing indicated that RNA editing can produce proteins that are highly conserved among plant species.

RNA editing commonly occurs at particular positions with bases bias [22]. In Catharanthus atp genes, editing events also like the second position codon in most. It has been proposed that C-to-U RNA editing was a specific procedure for protection in the evolution of plants [23]. The results indicate that the second site of the codon has the highest editing recurrence, up to $80 \%$ of the total editing sites except one in the third codon. The highest frequent RNA editing has been documented in the second position of the affected codon, followed by the first position, and not many cases occur in the third position [30].

RNA editing events could switch hydrophilic amino acids to hydrophobic amino acids and occur at positions necessary for protein reaction [15]. The preserved RNA editing sites have their codon predilection, leading to a dissimilar substitution frequency of amino acids and the generality substitution residue is serine to leucine [22]. Hydrophobic amino acid codons ratio is increased after RNA editing [32] and that may stimulate hydrophobicity of resultant proteins which is essential for enzyme function in plastid complex [33]. Hydrophobic amino acids and the presence of leucine, in particular, can be participated in binding/recognition of hydrophobic ligands such as lipids [34]. For each of the two edited genes, the cDNA transcripts in the different tissues were aligned with the reference genes using CLC genomic workbench to analyze affected amino acids. Bioinformatics analysis demonstrated that four editing sites, atpA-773, atpA-791, atpA-914 and atpI-620 in two genes of the ATP complex change the corresponding amino acid sequences from ser to leu because of RNA editing. Therefore, converted amino acids increased hydrophobicity in the final products which could induce its function. Interestingly, a comparative analysis of converted amino acids among the various tissues after editing demonstrated that all these sites found in all studied tissues unless atpA-914 was missing in flower. These modifications suggest that the mentioned above editing events might have an effect on the action of produced proteins. 


\section{CONCLUSION}

In the present investigation, we detected RNA editing events in ATP genes complex among six tissues of $C$. roseus chloroplast genome using bioinformatics technique. It has been detected five edited sites in only two genes (atpA and atpI) of the six examined genes. Four edited sites led to codon conversions identifying different amino acids and one had no effect on the corresponding amino acid. All the four amino acid conversions being were Ser-Luc which could motivate the hydrophobicity and may increase the efficiency of affected proteins. Taken together, the outputs implied that RNA editing in the plastid ATP complex could impact protein structure which in turn affects its function. However, whether these edited sites play a key role in the photosynthetic efficiency in $C$. roseus tissues needs further consideration.

\section{REFERENCES}

[1] Takenaka, M., Zehrmann, A., Verbitskiy, D., Härtel, B., \& Brennicke, A. (2013). RNA Editing in Plants and Its Evolution. Annual Review of Genetics, 47(1), 335-352.doi:10.1146/annurev-genet-111212-133519

[2] Meng Y, Chen D, Jin Y, Mao C, Wu P, Chen M (2010) RNA editing of nuclear transcripts in Arabidopsis thaliana. BMC Genomics 11:1-7

[3] Sun, T., Bentolila, S., \& Hanson, M. R. (2016). The Unexpected Diversity of Plant Organelle RNA Editosomes. Trends in Plant Science, 21(11), 962-973.doi:10.1016/j.tplants.2016.07.005

[4] Lurin C, Andrés C, Aubourg S, Bellaoui M, Bitton F, Bruyère C, Caboche M, Debast C, Gualberto J, Hoffmann B, Lecharny A, Le Ret M, Martin-Magniette ML, Mireau H, Peeters N, Renou JP, Szurek B, Taconnat L, Small I (2004) Genome-wide analysis of Arabidopsis pentatricopeptide repeat proteins reveals their essential role in organelle biogenesis. Plant Cell 16:2089-2103

[5] Doniwa, Y., Ueda, M., Ueta, M., Wada, A., Kadowaki, K.I. and Tsutsumi, N. (2010) The involvement of a PPR protein of the P subfamily in partial RNA editing of an Arabidopsis mitochondrial transcript. Gene, 454, 39-46.

[6] Chateigner-Boutin, A., \& Small, I. (2010). RNA Biology: Plant RNA editing (Vol. 7:2, 213-219). Crawley, Australia: Australian Research Council Centre of Excellence in Plant Energy Biology; University of Western Australia.

[7] Leu, K.C., Hsieh, M.H., Wang, H.J., Hsieh, H.L. and Jauh, G.Y. (2016) Distinct role of Arabidopsis mitochondrial P-type pentatricopeptide repeat protein-modulating editing protein, PPME, in nad1 RNA editing. RNA Biol. 13, 593-604.

[8] Wagoner JA, Sun T, Lin L, Hanson MR (2015) Cytidine deaminase motifs within the DYW domain of Two pentatricopeptide repeatcontaining proteins are required for site-specific chloroplast RNA editing. J Biol Chem 290:2957-2968

[9] Guillaumot D, Lopez-Obando M, Baudry K, Avon A, Rigaill G, Falcon de Longevialle A, Broche B, Takenaka M, Berthomé R, De Jaeger G, Delannoy E, Lurin C (2017) Two interacting PPR proteins are major Arabidopsis editing factors in plastid and mitochondria. Proc Natl Acad Sci USA 114:8877-8882

[10] Shikanai T (2015) RNA editing in plants: Machinery and flexibility of site recognition. Biochim Biophys Acta 1847:779785

[11] Cheng S, Gutmann B, Zhong X, Ye Y, Fisher MF, Bai F, Castleden I, Song Y, Song B, Huang J, Liu X, Xu X, Lim BL, Bond CS, Yiu SM, Small I (2016) Redefining the structural motifs that determine RNA binding and RNA editing by pentatricopeptide repeat proteins in land plants. Plant J 85:532-547

[12] Tseng CC, Lee CJ, Chung YT, Sung TY, Hsieh MH (2013) Differential regulation of Arabidopsis plastid gene expression and RNA editing in non-photosynthetic tissues. Plant J 82:375-392

[13] Qulsum, U., Azad, M. T. A., \& Tsukahara, T. (2019). Analysis of Tissue-specific RNA Editing Events of Genes Involved in RNA Editing in Arabidopsis thaliana. Journal of Plant Biology, 62(5), 351-358. doi:10.1007/s12374-018-0452-5

[14] Barkan A, Small I (2014) Pentatricopeptide repeat proteins in plants. Annu Rev Plant Biol 65:415-442

[15] Yura, Kei\& Go, Mitiko (2008). Correlation between amino acid residues converted by RNA editing and functional residues in protein three-dimensional structures in plant organelles. BMC Plant Biology. doi.org/10.1186/1471-2229-8-79

[16] Jarvis, Paul, López-Juez, Enrique. (2013). Biogenesis and homeostasis of chloroplasts and other plastids. Nature Reviews Molecular Cell Biology. 14, 787-802. doi.org/10.1038/nrm3702

[17] Peltier, J. B., Friso, G., Kalume, D. E., Roepstorff, P., Nilsson, F., Adamska, I., \& van Wijk, K. J. (2000). Proteomics of the chloroplast: systematic identification and targeting analysis of lumenal and peripheral thylakoid proteins. The Plant cell. 12(3), 319-41.

[18] Hahn, A., Vonck, J., Mills, D. J., Meier, T. \& Kühlbrandt, W. (2018). Structure, mechanism, and regulation of the chloroplast ATP synthase. Science 360, eaat 4318 .

[19] Chen, F., Dong, G., Wu, L., Wang, F., Yang, X., Ma, X., ... Yu, Y. (2016). A Nucleus-Encoded Chloroplast Protein YL1 Is Involved in Chloroplast Development and Efficient Biogenesis of Chloroplast ATP Synthase in Rice. Scientific reports, 6, 32295. doi:10.1038/srep32295

[20] Von Ballmoos, C., Wiedenmann, A., \& Dimroth, P. (2009). Essentials for ATP Synthesis by F1F0ATP Synthases. Annual Review of Biochemistry, 78(1), 649-672. doi: 10.1146/annurev.biochem.78.081307.10480

[21] Tillich, M. , Funk, H. T., Schmitz-Linneweber, C. , Poltnigg, P. , Sabater, B. , Martin, M. and Maier, R. M. (2005). Editing of plastid RNA in Arabidopsis thaliana ecotypes. The Plant Journal, 43: 708-715. doi:10.1111/j.1365-313X 
[22] Jiang Y, Fan SL, Song MZ, Yu JN, Yu SX. (2012). Identification of RNA editing sites in cotton (Gossypium hirsutum) chloroplasts and editing events that affect secondary and three-dimensional protein structures. Genet Mol Res, 11(2):987-1001. doi:10.4238

[23] Häder DP and Sinha RP (2005). Solar ultraviolet radiation-induced DNA damage in aquatic organisms: potential environmental impact. Mutat. Res. 571: 221-233.

[24] Maier, R. M., Neckermann, K., Igloi, G. L., \& Kössel, H. (1995). Complete Sequence of the Maize Chloroplast Genome: Gene Content, Hotspots of Divergence and Fine Tuning of Genetic Information by Transcript Editing. Journal of Molecular Biology, 251(5), 614-628. doi:10.1006/jmbi.1995.0460

[25] Hirose, T., Kusumegi, T., Tsudzuki, T., \& Sugiura, M. (1999). RNA editing sites in tobacco chloroplast transcripts: editing as a possible regulator of chloroplast RNA polymerase activity. Molecular and General Genetics MGG, 262(3), 462467. doi:10.1007/s004380051106 )

[26] Tsudzuki, T., Wakasugi, T., \& Sugiura, M. (2001). Comparative Analysis of RNA Editing Sites in Higher Plant Chloroplasts. Journal of Molecular Evolution, 53(4-5), 327-332. doi:10.1007/s002390010222

[27] Inada, M., Sasaki, T., Yukawa, M., Tsudzuki, T. and Sugiura, M. (2004). A systematic search for RNA editing sites in pea chloroplast: an editing event causes diversification from the evolutionarily conserved amino acid sequence. Plant cell physiol. 45, 1615-1622.

[28] Corneille, S., Lutz, K., \& Maliga, P. (2000). Conservation of RNA editing between rice and maize plastids: are most editing events dispensable? Molecular and General Genetics MGG, 264(4), 419-424. doi:10.1007/s004380000295

[29]Zeng, W. h., Liao, S. C., Chang, C.C. (2007). Identification of RNA Editing Sites in Chloroplast Transcripts of Phalaenopsis aphrodite and Comparative Analysis with Those of Other Seed Plants , Plant and Cell Physiology, 48(2):362-368, https://doi.org/10.1093/pcp/pc1058)

[30] Wolf PG, Rowe CA and Hasebe M (2004). High levels of RNA editing in a vascular plant chloroplast genome: analysis of transcripts from the fern Adiantum capillus-veneris. Gene 339: 89-97.

[31] Bock R (2000). Sense from nonsense: how the genetic information of chloroplasts is altered by RNA editing. Biochimie 82: 549-557.

[32] Giege' P. and A.Brennicke (1999). RNA editing in Arabidopsis mitochondria affects 441 C to U changes in ORFs. ProcNatlAcadSci U S A. 96(26): 15324-15329.

[33] Kalinati Y.N., V. Dinesh Kumar, S.S. Reddy. (2008). RNA editing in NAD3 and ATP9 transcripts of safflower (Carthamustinctorius). International Journal of Biology. 3:143-149.

[34] Matthew J. Betts, Robert B. Russell, Michael R. Barnes and Ian C. Gray (2003). Amino Acid Properties and Consequences of Substitutions. Bioinformatics for Geneticists. John Wiley \& Sons, Ltd. ISBNs: 0-470-84393-4 (HB); 0-470-84394-2 (PB).

[35] Wang, M., Liu, H., Ge, L., Xing, G., Wang, M., Weining, S., \& Nie, X. (2016). Identification and Analysis of RNA Editing Sites in the Chloroplast Transcripts of Aegilops tauschii L. Genes, 8(1), 13. doi:10.3390/genes8010013 